BASIC SCIENCE

\title{
Androgen Receptor (AR) Gene (CAG)n and (GGN)n Length Polymorphisms and Symptoms in Young Males With Long-Lasting Adverse Effects After Finasteride Use Against Androgenic Alopecia
}

Sabina Cauci, $\mathrm{PhD},{ }^{1}$ Giovanni Chiriacò, MD, ${ }^{2}$ Erika Cecchin, $\mathrm{MS}_{1}{ }^{3}$ Giuseppe Toffoli, MD, ${ }^{3}$ Serena Xodo, MD, Giuseppe Stinco, $\mathrm{MD}^{5}$ and Carlo Trombetta, $\mathrm{MD}^{2}$

\section{ABSTRACT}

Introduction: Long-term adverse symptoms of men who used oral finasteride against androgenic alopecia have been recently described as post-finasteride syndrome (PFS).

Aim: To determine whether (CAG)n-rs4045402 and (GGN)n-rs3138869 polymorphisms in the androgen receptor $(A R)$ gene are implicated in PFS.

Methods: $A R$ polymorphisms were studied according to PFS symptoms in 66 white participants (31.8\% Italian, $28.8 \%$ American, and $39.4 \%$ other).

Main Outcome Measures: Symptoms were investigated by an ad hoc 100-item questionnaire and the Arizona Sexual Experience Scale and Aging Male Symptom Scale (AMS). (CAG)n and (GGN)n repeats were categorized as short ([CAG]9-19, [GGN]<23), medium ([CAG]20-24, [GGN]23), or long ([CAG]25-37, [GGN]>23).

Results: Median age was 32 years, duration of finasteride use was 360 days, and time from finasteride discontinuation was 1,053 days. We observed several frequency differences in symptoms according to (CAG)n and (GGN)n repeat numbers. Three AMS items were worse for medium (GGN)23 than for long (GGN) $>23$ carriers and one item was worse for short $(\mathrm{GGN})<23$ carriers. The AMS item for decrease in sexual desire or libido was worse for short (CAG)9-19 carriers than for medium (CAG)20-24 carriers. Through the ad hoc questionnaire, significant findings in (CAG)n and/or (GGN)n repeats were obtained for penile discomfort, loss of scrotal sensitivity, scrotal discomfort, less pubic hair, loss of perceived perineal fullness, increased sperm density, involuntary muscle spasms, loss of muscle tone, increased weight $(>2 \mathrm{~kg})$, increased skin dryness, and onset of symptoms after finasteride use.

Conclusion: This study showed that short and/or long (CAG)n and (GGN)n repeats had different frequencies according to symptoms reported by patients with PFS, likely reflecting the vast array of genes modulated by the AR. This study showed a U-curvilinear profile of (CAG)n repeats for skin dryness symptoms, where the two extremes exhibited a worse condition than medium repeats. Further studies are necessary to investigate the PFS pathophysiology using a precision medicine approach. Cauci S, Chiriacò G, Cecchin E, et al. Androgen Receptor (AR) Gene (CAG)n and (GGN)n Length Polymorphisms and Symptoms in Young Males With Long-Lasting Adverse Effects After Finasteride Use Against Androgenic Alopecia. Sex Med 2017;5:e61-e71.

Copyright $@ 2$ 2016, The Authors. Published by Elsevier Inc. on behalf of the International Society for Sexual Medicine. This is an open access article under the CC BY-NC-ND license (http://creativecommons.org/licenses/by-nc-nd/4.0/).

Key Words: $5 \alpha$-Reductase Inhibitor; Post-Finasteride Syndrome; Male Pattern Hair Loss; Androgenic Alopecia; Androgen Receptor; CAG Polymorphism; GGN Polymorphism; Erectile Dysfunction; Sexual Dysfunction; Loss of Libido; Finasteride Side Effects; Finasteride Safety

Received September 1, 2016. Accepted November 10, 2016.

'Department of Medical and Biological Sciences, School of Medicine, University of Udine, Udine, Italy;

${ }^{2}$ Urological Hospital Department, Department of Medical, Surgical and Health Sciences, University of Trieste, Trieste, Italy;

${ }^{3}$ Experimental and Clinical Pharmacology Unit, CRO Aviano National Cancer Institute, Italy;

${ }^{4}$ Hospital Department of Gynecology and Obstetrics, University Hospital Santa Maria della Misericordia, Udine, Italy;
${ }^{5}$ Department of Experimental and Clinical Medicine, University of Udine, Dermatology Clinic, University Hospital Santa Maria della Misericordia, Udine, Italy

Copyright (c) 2016, The Authors. Published by Elsevier Inc. on behalf of the International Society for Sexual Medicine. This is an open access article under the CC BY-NC-ND license (http://creativecommons.org/ licenses/by-nc-nd/4.0/).

http://dx.doi.org/10.1016/j.esxm.2016.11.001 


\section{INTRODUCTION}

Recent studies have described severe adverse effects in young men who used oral finasteride against androgenic alopecia (AGA) that persisted several months or years after finasteride discontinuation $^{1-5}$ (a condition called post-finasteride syndrome $\left.[\mathrm{PFS}]^{4,6}\right)$. A meta-analysis of clinical trials of finasteride on subjects with AGA showed that the toxicity information was very limited, of poor quality, and likely to be systematically biased.'

Finasteride inhibits $5 \alpha$-reductase, the enzyme responsible for the reduction of testosterone into dihydrotestosterone. ${ }^{5}$ Finasteride against AGA (male pattern hair loss) is used at lower dosage $(1 \mathrm{mg} / \mathrm{d})$ than against benign prostatic hyperplasia $(5 \mathrm{mg} / \mathrm{d})$. Finasteride inhibits $5 \alpha$-reductase type 2 and 3 enzymes much more strongly than the type 1 enzyme ${ }^{8,9}$; therefore, finasteride can affect several different human tissues, ${ }^{4,5,8,10}$ such as the prostate, muscle, liver, kidney, brain, mammary gland, frontal cortex, skin, epidermis, pancreas, spleen, heart, testicle, stomach, dermis, small intestine, and adipose tissues. ${ }^{8,9,11}$

Finasteride use has several adverse effects, including erectile dysfunction, loss of libido, and smaller ejaculatory volume., , $10,12^{-12}$ A meta-analysis on the effects of $5 \alpha$-reductase inhibitors found a significant pooled relative risk for sexual dysfunction in men with benign prostatic hyperplasia $(2.56,95 \% \mathrm{CI}=1.48-4.42)$ but no significant increased risk in men with AGA (1.21, 95\% $\mathrm{CI}=0.85-1.72){ }^{13}$

Recently, a clinical study described the main symptoms of subjects with PFS, including loss of penis sensitivity, decreased ejaculatory force, low penile temperature, smaller ejaculatory volume, anhedonia, lack of mental concentration, and loss of muscle tone or mass. ${ }^{4}$ In particular, an immunohistochemical study found increased levels of the androgen receptor (AR) in epithelial and stromal cells from the foreskin of eight men with PFS compared with healthy men. ${ }^{14}$

The expression level and amino acid protein sequence of $\mathrm{AR}$ can be affected by polymorphisms in its gene $(A R) .{ }^{15}$ The most frequently studied polymorphisms of $A R$ are two repeated nucleotide sequences: the (CAG)nCAA repeat nucleotide sequence, denoted as $(\mathrm{CAG}) \mathrm{n}$, encoding a polyglutamine stretch, and the polymorphic repeat (CGT) ${ }_{3} \mathrm{GGG}(\mathrm{GGT})_{2}(\mathrm{GGC}) \mathrm{n}$, denoted as (GGN)n, encoding a poly-glycine stretch. The two polymorphisms are included in the $\mathrm{N}$-terminal of the AR protein and compose the transactivation domain of the nuclear receptor. ${ }^{16}$

The (CAG)n repeat length usually spans 9 to 36 repeat units, although the number varies among ethnic groups. ${ }^{17}$ Long (CAG) $\mathrm{n}$ repeats have been associated with decreased AR transactivation activity and weaker transcriptional potential than short repeats. ${ }^{18}$ CAG expanded repeats of at least 40 have been found in Kennedy disease, a neurodegenerative syndrome also characterized by androgen insensitivity. ${ }^{18-20}$ Long (CAG)n repeats have been associated with male infertility, ${ }^{21}$ although studies have been inconsistent. ${ }^{17,18,21}$ In contrast, a meta-analysis suggested that a shorter $(\mathrm{CAG}) \mathrm{n}$ repeat polymorphism in Caucasians and
Asians might increase the risk of prostate cancer compared with the longer (CAG)n repeat. ${ }^{22}$

The trinucleotide (GGN)n has been less investigated than the $(\mathrm{CAG}) \mathrm{n}$ repeat polymorphism with respect to male androgenicity and infertility. Moreover, studies have not been very consistent. $^{21}$ In an in vitro study, (GGN)23 showed higher transcription than shorter or longer repeats. ${ }^{23}$ A meta-analysis found a correlation of long (GGN) $\geq 23$ with testicular cancer. ${ }^{24}$

A recent molecular study (of 69 men with AGA and PFS, 91 men with untreated AGA, and 78 healthy men without AGA) focused on whether the two polymorphisms, (CAG)n-rs4045402 and (GGN)n-rs3138869, in the $A R$ gene might play a role in the toxic long-term effects of finasteride. ${ }^{6}$ This study suggested that extreme repeats are a genetic predisposing factor for AGA development.

However, the pathophysiology of PFS remains largely unknown and detailed molecular events predisposing to specific long-term symptoms experienced by patients with PFS remain obscure. 5

In our previous genetic study, ${ }^{6}$ we did not examine the relation of $A R(\mathrm{CAG}) \mathrm{n}$ and (GGN)n polymorphisms with the single specific symptoms of subjects with PFS. In the present study, we explored this relation by three different questionnaires-the Arizona Sexual Experience Scale (ASEX), ${ }^{25}$ the Aging Male Symptom Scale (AMS), ${ }^{26}$ and our ad hoc 100-item questionnaire ${ }^{4}$-for the clinical symptoms of 66 men with PFS. We also collected retrospectively the genetic data from our previous study ${ }^{6}$ to check whether less common repeat lengths of (CAG)n-rs4045402 and (GGN)n-rs3138869 polymorphisms might be related to the specific symptoms described by subjects with PFS.

\section{METHODS}

\section{Subjects}

Enrollment, inclusion, and exclusion criteria were previously described. ${ }^{4,6}$ Obese subjects (body mass index $>30.0 \mathrm{~kg} / \mathrm{m}^{2}$ ) were excluded from this study because of the relation of fat body composition to androgens. ${ }^{27}$ According to the inclusion criteria, all participants were white. ${ }^{28}$ Moreover, because of the location of $A R$ in the $\mathrm{X}$ chromosome, and to further confirm race, each participant was specifically asked to declare whether he had a white mother. ${ }^{6}$ None of the subjects declared homo- or bisexuality. The institutional ethical committee of each participating institution approved the study protocol (according to the Declaration of Helsinki), and all subjects signed a written informed consent.

This was an observational and retrospective study. We enrolled men ( $\geq 18$ and $\leq 50$ years old) who used oral finasteride for AGA and had developed persistent adverse effects lasting for at least 6 months after drug discontinuation. ${ }^{4,6}$ Of 69 men initially enrolled, ${ }^{6}$ three were excluded for incomplete 
questionnaires, leaving 66 subjects with PFS to be examined. Finasteride was used orally mostly at the dose of $1 \mathrm{mg} / \mathrm{d}$, but some patients (to save money) broke a 5-mg pill into four parts $(\sim 1.25 \mathrm{mg} / \mathrm{d})$ or broke a $1-\mathrm{mg}$ pill into two parts $(\sim 0.5 \mathrm{mg} / \mathrm{d})$.

\section{Assessment of Symptoms and AR Polymorphisms}

Three different questionnaires were used to evaluate adverse effects persisting longer than 6 months. We developed an ad hoc 100-item questionnaire to interview subjects with PFS about their demographic and clinical characteristics. ${ }^{4}$ In addition, participants filled out the $\mathrm{ASEX}^{25}$ reporting on their condition at the time of study (current ASEX) and retrospectively in addressing how they were before finasteride use (pre-ASEX) to rule out any sexual dysfunction before they started to use finasteride. However, because of possible recall bias for data before finasteride use, the pre-ASEX score was not used further to analyze the role of $A R$ polymorphisms. The ASEX consists of five items with rating scales graded from 1 to 6 that quantify sex drive, arousal, penile erection, ability to reach orgasm, and satisfaction from orgasm, with higher scores indicating more severe sexual dysfunction.

Furthermore, participants filled out the 17-item AMS assessing androgenic dysfunction ${ }^{26,29}$ by three subscales for psychological, somatic, and sexual symptoms. Each item is graded from 1 (absent) to 5 (very severe); the total AMS score defines androgen deficiency as absent (score $=17-26)$, slight (score $=$ $27-36$ ), moderate (score $=37-49$ ), or severe $($ score $\geq 50)$. ${ }^{26}$

The present inclusion criteria were a current ASEX total score corresponding to sexual dysfunction (total ASEX score $\geq 19$ or any one item score $=5$ or any three items with score $\geq 4)^{25}$ and/or AMS total score of least 27. ${ }^{4}$ Exclusion criteria included an ASEX score of at least 19 before finasteride use. ${ }^{4}$

For assessment of $A R(\mathrm{CAG}) \mathrm{n}$ and (GGN)n polymorphisms, genomic DNA was extracted from blood or saliva samples, amplified, and sequenced as described previously. ${ }^{6}$

\section{Statistical Analysis}

$A R(\mathrm{CAG}) \mathrm{n}$ and $(\mathrm{GGN}) \mathrm{n}$ repeat lengths were divided into three categories-low quartile ( $<25$ th percentile, short repeats), interquartile (25th to 75 th percentile, medium repeats), and high quartile ( $>75$ th percentile, long repeats) —and analyzed in a binary logistic regression model. For (GGN)n repeats, the central (GGN) 23 repeat was found in more than $50 \%$ of subjects ( 44 of $66,67 \%)$; therefore, short repeats $(\mathrm{GGN})<23$ and long repeats (GGN) $>23$ necessarily had a frequency lower than $25 \%$.

The Kolmogorov-Smirnov test was adopted to assess the normal data distribution. Continuous data not normally distributed were expressed as median (25th to 75th percentile, interquartile range) and comparisons between groups were performed by Mann-Whitney U-test. Odds ratios (ORs) and 95\% CIs were calculated to assess relative risks for binary (yes or no) variables, and the Pearson $\chi^{2}$ or Fisher test was used to calculate the $P$ value, as appropriate. Two-sided $P$ values less than .05 were considered significant $(P<.10$ indicated a tendency). SPSS for Windows (IBM Corp, Armonk, NY, USA) was used.

\section{RESULTS}

Demographic characteristics and results from the ad hoc questionnaire, ASEX, and AMS are presented in Table 1. Subjects were enrolled a median of nearly 3 years after finasteride discontinuation and had used finasteride for 12 months.

Distribution of (CAG)n and (GGN)n repeat frequencies in the 66 subjects with PFS is illustrated in Supplementary Figures S1 and S2, respectively. Frequencies of subjects with short, medium, and long repeat lengths for the two polymorphisms were categorized: short $=(\mathrm{CAG}) 9-19(\mathrm{n}=18,27.3 \%)$ and $(\mathrm{GGN})<23(\mathrm{n}=8$, $12.1 \%) ;$ medium $=(\mathrm{CAG}) 20-24(\mathrm{n}=37,56.1 \%)$ and $(\mathrm{GGN})$ $23(\mathrm{n}=44,66.7 \%)$; long $=(\mathrm{CAG}) 25-37(\mathrm{n}=11,16.7 \%)$ and $(\mathrm{GGN})>23(\mathrm{n}=14,21.2 \%)$.

We did not find significant differences among the subgroups of (CAG)n and (GGN)n repeat lengths for age at enrollment, age at starting drug use, duration of finasteride use, period from drug discontinuation to study enrollment, and body mass index (Table 2). However, we observed a decreasing trend for duration of finasteride use and decrease of $(\mathrm{CAG}) \mathrm{n}$ repeats with median values of 620 days for long (CAG)25-37, 450 days for medium (CAG)20-24, and 180 days for short (CAG)9-19 (short repeats showed a tendency to differ from medium repeats $[P=.062]$, but the difference for short [CAG]9-19 vs medium to long $[C A G] \geq 20$ was significant $[P=.045])$.

Table 2 presents findings from the ASEX and AMS. The total scores of the ASEX and AMS did not differ with length of $(\mathrm{CAG}) \mathrm{n}$ and (GGN)n repeats; however, we found differences for single items. For clarity, Table 2 presents only symptom items that showed a median number of points that differed statistically between at least two repeat length groups. ASEX item 5 (orgasm satisfaction) was worse in the long (CAG) 25-37 than in the short (CAG)9-19 subgroup $(P=.040)$. Five AMS items showed differences among $(C A G) n$ and/or (GGN)n subgroups. AMS item 5 (increased need for sleep and/or often feeling tired) was higher in the medium (GGN)23 than in the short $(\mathrm{GGN})<23$ group $(P=.048)$. AMS items 9 (physical exhaustion or lacking vitality), 11 (depressive mood), and 12 (feeling that one has passed one's peak) were worse in the medium (GGN) 23 than in the long $(\mathrm{GGN})>23$ subgroup $(P=.042, P=.036$, and $P=.044$, respectively). In contrast, AMS item 17 (decrease in sexual desire/libido) was worse in the short (CAG)9-19 than in the medium (CAG)20-24 group $(P=.028)$

Tables 3 and 4 present data collected by the ad hoc questionnaire. Data were reported as binary variables, and only symptom items with at least one significant finding in relation to $(\mathrm{CAG}) \mathrm{n}$ and/or $(\mathrm{GGN}) \mathrm{n}$ repeats, respectively, were indicated.

Table 3 presents significant findings in relation to $(C A G) n$ repeats. Scrotal discomfort was less frequent in the short 
Table 1. Demographic characteristics, finasteride use, and symptoms of 66 subjects with post-finasteride syndrome

\begin{tabular}{|c|c|}
\hline $\begin{array}{l}\text { Age (y), median (25th-75th } \\
\text { percentile), range }\end{array}$ & 32 (27-39), 21-50 \\
\hline $\begin{array}{l}\text { BMl }\left(\mathrm{kg} / \mathrm{m}^{2}\right) \text {, median }(25 \mathrm{th}-75 \text { th } \\
\text { percentile), range }\end{array}$ & $\begin{array}{l}23.9(22.4-26.2) \\
17.3-29.9\end{array}$ \\
\hline \multicolumn{2}{|l|}{ Nationality, n (\%) } \\
\hline Italy & $21(31.8)$ \\
\hline United States & 19 (28.8) \\
\hline Canada & 9 (13.6) \\
\hline United Kingdom & $6(9.1)$ \\
\hline France & $3(4.5)$ \\
\hline Spain & $2(3.0)$ \\
\hline Bulgaria & $1(1.5)$ \\
\hline Hungary & $1(1.5)$ \\
\hline Sweden & $1(1.5)$ \\
\hline Australia & $1(1.5)$ \\
\hline Brazil & $1(1.5)$ \\
\hline Israel & $1(1.5)$ \\
\hline \multicolumn{2}{|l|}{ Educational level, n (\%) } \\
\hline Elementary school & $1(1.5)$ \\
\hline High school & 15 (22.7) \\
\hline College or university & 50 (75.8) \\
\hline \multicolumn{2}{|l|}{ Marital status, n (\%) } \\
\hline Single & $54(81.8)$ \\
\hline Married & 9 (13.6) \\
\hline Divorced & $3(4.5)$ \\
\hline $\begin{array}{l}\text { Age at starting finasteride }(y) \text {, } \\
\text { median ( } 25 \text { th }-75 \text { th } \\
\text { percentile), range }\end{array}$ & $26(22-31), 18-48$ \\
\hline $\begin{array}{l}\text { Duration of finasteride use } \\
\text { (d), median ( } 25 \text { th-75th } \\
\text { percentile), range }\end{array}$ & $\begin{array}{l}360(163-1,298) \\
17-3,650\end{array}$ \\
\hline $\begin{array}{l}\text { Discontinuation of finasteride } \\
\text { (d), median ( } 25 \text { th- }-75 \text { th } \\
\text { percentile), range }\end{array}$ & $\begin{array}{l}1,053(560-2,043) \\
181-5,057\end{array}$ \\
\hline \multicolumn{2}{|l|}{ Dosage used, n (\%) } \\
\hline $1 \mathrm{mg} / \mathrm{d}$ & $46(69.7)$ \\
\hline $1.25 \mathrm{mg} / \mathrm{d}$ & $16(24.2)$ \\
\hline 0.5 mg/d & $4(6.1)$ \\
\hline \multicolumn{2}{|l|}{ Onset of symptoms, n (\%) } \\
\hline During finasteride use & 59 (89.4) \\
\hline After finasteride use & $7(10.6)$ \\
\hline$\leq 1 \mathrm{mo}$ after discontinuation & $5(7.6)$ \\
\hline$>1$ mo after discontinuation & $2(3.0)$ \\
\hline \multicolumn{2}{|l|}{$\begin{array}{l}\text { Trend of symptoms after } \\
\quad \text { finasteride discontinuation, } \mathrm{n}(\%)\end{array}$} \\
\hline Worsening & 38 (57.6) \\
\hline Unchanged & 19 (28.8) \\
\hline Improved & 9 (13.6) \\
\hline \multicolumn{2}{|l|}{ Sexual symptoms, n [\%]* } \\
\hline Loss of penis sensitivity & 58 (87.9) \\
\hline Decreased ejaculatory force & 54 (81.8) \\
\hline Decreased penile temperature & $49(74.2)$ \\
\hline Decreased ejaculate volume & 47 (71.2) \\
\hline Loss of scrotum fullness & $45(68.2)$ \\
\hline
\end{tabular}

(continued)
Table 1. Continued

\begin{tabular}{|c|c|}
\hline Penile flaccidity or wrinkled & $42(63.6)$ \\
\hline Decrease of penile dimension & $42(63.6)$ \\
\hline Loss of scrotum sensitivity & 41 (62.1) \\
\hline \multicolumn{2}{|l|}{ Mental disorders, n (\%)* } \\
\hline $\begin{array}{l}\text { Decreased pleasure in life or } \\
\text { emotions (anhedonia) }\end{array}$ & 51 (77.3) \\
\hline Lack of mental concentration & $49(74.2)$ \\
\hline \multicolumn{2}{|l|}{ Somatic symptoms, n (\%)* } \\
\hline Loss of muscle tone or mass & $34(51.5)$ \\
\hline $\begin{array}{l}\text { AMS total score (points), median } \\
\text { (25th-75th percentile), range }\end{array}$ & 52 (44-61), 29-75 \\
\hline $\begin{array}{l}\text { Somato-vegetative } \\
\text { subscale (points) }\end{array}$ & $19(16-24), 9-30$ \\
\hline Psychological subscale (points) & 15 (11-19), 5-25 \\
\hline Sexual subscale (points) & 19 (16-21), 10-23 \\
\hline $\begin{array}{l}\text { AMS total score }<37 \text { points } \\
\text { (slight clinical androgen } \\
\text { deficiency), } \mathrm{n}(\%)\end{array}$ & $3(4.5)$ \\
\hline $\begin{array}{l}\text { AMS total score } \geq 50 \text { points } \\
\text { (severe clinical androgen } \\
\text { deficiency), } \mathrm{n}(\%)\end{array}$ & 38 (57.6) \\
\hline $\begin{array}{l}\text { Current ASEX total score (points), } \\
\text { median ( } 25 \text { th-75th } \\
\text { percentile), range }\end{array}$ & $22(19-23), 15-30^{\dagger}$ \\
\hline $\begin{array}{l}\text { Current ASEX total score } \\
\geq 19 \text { points (severe sexual } \\
\text { dysfunction), } \mathrm{n}(\%)\end{array}$ & $52(78.8)$ \\
\hline $\begin{array}{l}\text { ASEX total score before } \\
\text { finasteride use (points), } \\
\text { median (25th-75th } \\
\text { percentile), range }\end{array}$ & $7(6-10), 5-15^{\dagger}$ \\
\hline $\begin{array}{l}\text { ASEX total score } \geq 19 \text { points } \\
\text { before finasteride use (severe sexual } \\
\text { dysfunction), } n(\%)\end{array}$ & $0(0.0)$ \\
\hline
\end{tabular}

AMS = Aging Male Symptom Scale; ASEX = Arizona Sexual Experience Scale; $\mathrm{BMI}=$ body mass index.

*Most frequent symptoms obtained from the authors' ad hoc 100-item questionnaire.

${ }^{\dagger} P<.001$, current ASEX score vs ASEX score before finasteride use.

(CAG)9-19 than in the medium (CAG)20-24 and medium to long $(\mathrm{CAG}) \geq 20$ groups $(33.3 \%, P=.027)$. Increase of sperm density was not reported by subjects with short (CAG)9-19 repeats but was noted by $40.5 \%$ of men with medium (CAG) $20-24(P=.001)$ repeats and by $35.4 \%$ of men with medium to long $(\mathrm{CAG}) \geq 20$ repeats $(P=.003)$. The ORs were 6.3 for involuntary muscle spasms in the long (CAG) $25-37$ vs medium (CAG) $20-24$ group and 5.5 vs the medium to short $(\mathrm{CAG})<25$ $(39.6 \%, P=.019)$ group. Increase of body weight $(>2 \mathrm{~kg})$ had an $\mathrm{OR}$ equal to 4.9 for the long (CAG)25-37 vs medium (CAG)20-24 group.

Notably, perceived increased skin dryness showed a curvilinear U-profile according to (CAG)n length. The medium 
Table 2. Comparison of subgroups of patients with post-finasteride syndrome according to AR gene (CAG)n and (CGN)n short, medium, and long repeats*

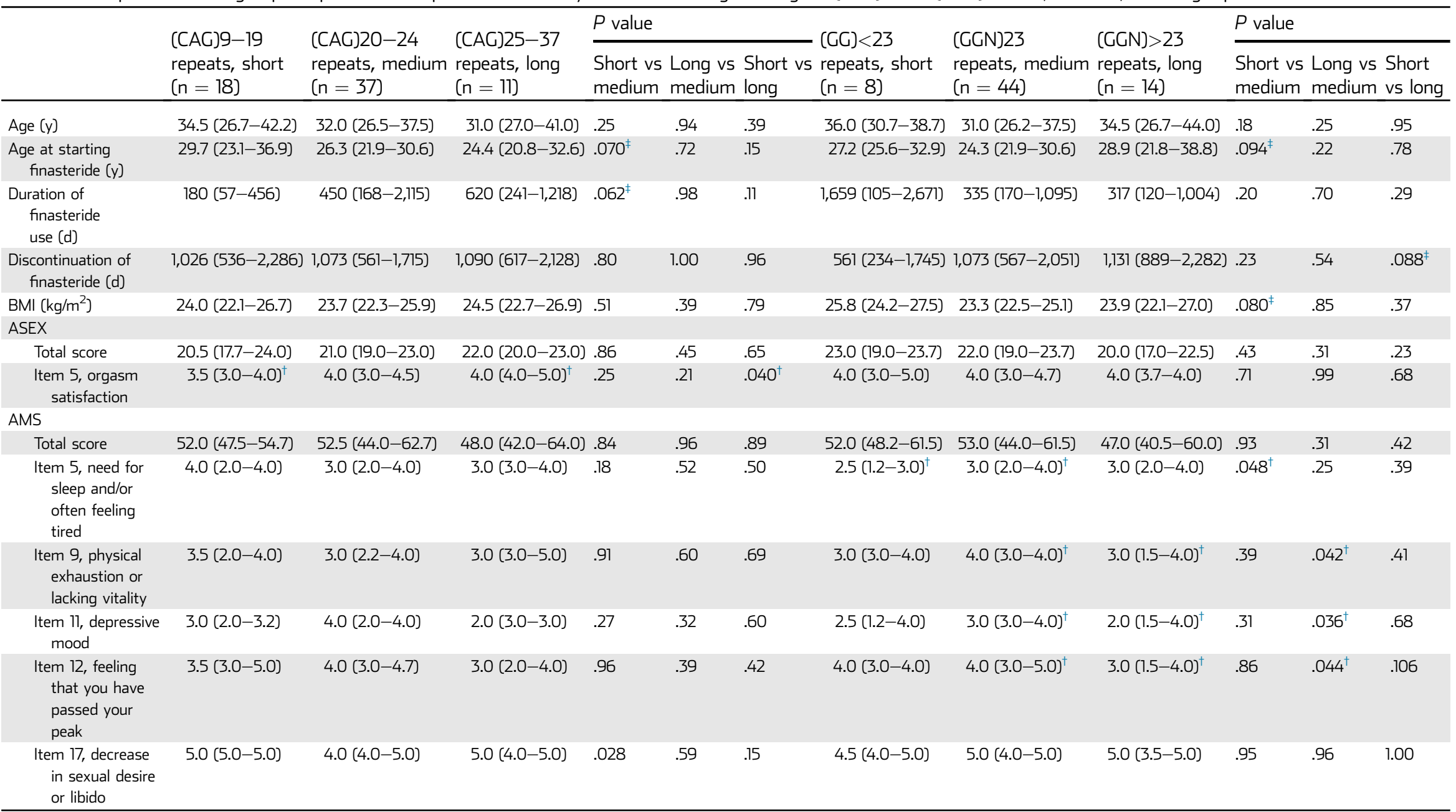

AMS = Aging Male Symptom Scale; ASEX = Arizona Sexual Experience Scale; BMI = body mass index.

*Continuous variables are reported as median (25th-75th percentile or interquartile range), and $P$ values were evaluated by two-tailed Mann-Whitney U-test.

†Significant differences.

†Nearly significant difference. 


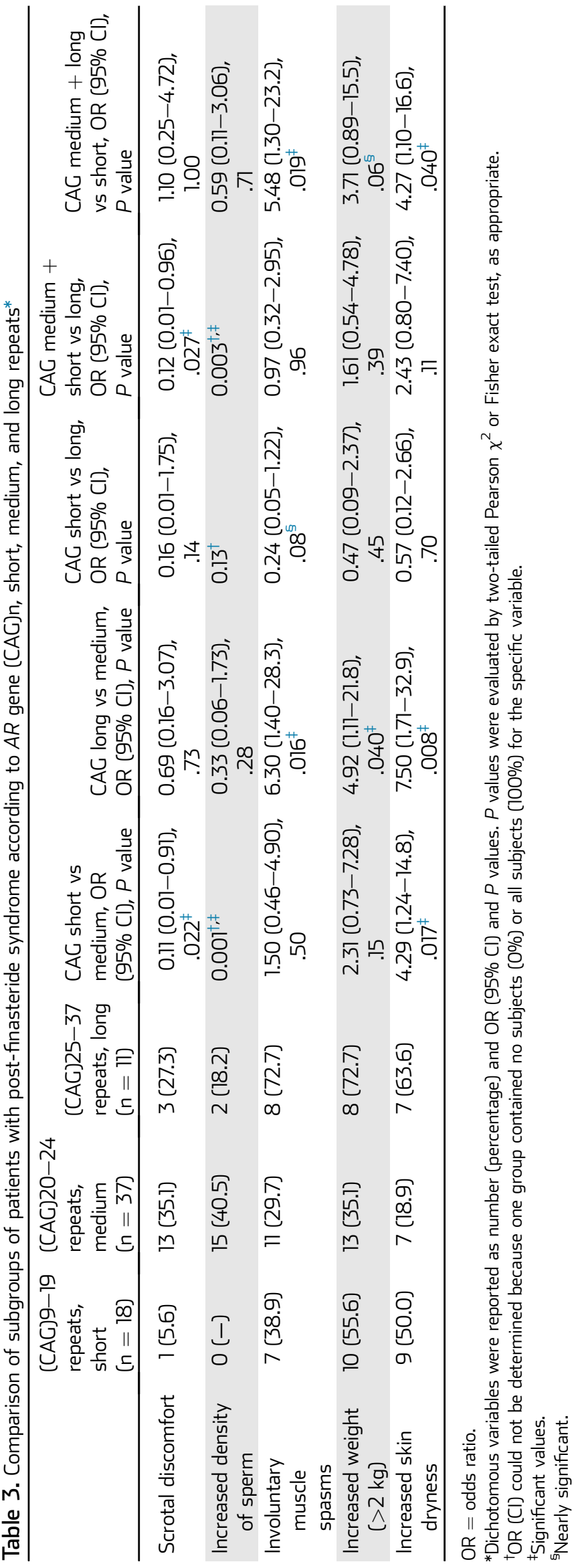

(CAG) 20-24 carriers had a lower frequency (18.9\%), whereas the short extreme (CAG)9-19 $(50.0 \%, \mathrm{OR}=4.3, P=.017)$ and long extreme (CAG) $25-37(63.6 \%, \mathrm{OR}=7.5, P=.008)$ carriers had higher frequencies.

Table 4 presents significant findings for (GGN)n repeats. Loss of scrotal sensitivity was less frequent in the long $(G G N)>23$ vs medium (GGN) 23 and medium to short $(\mathrm{GGN}) \leq 23(69.2 \%$, $P=.031)$ subgroups. No subject with long $(\mathrm{GGN})>23$ repeats reported scrotal discomfort vs $34.1 \%$ of subjects with medium $(\mathrm{GGN}) 23(P=.012)$ and $32.7 \%$ with medium to short $(\mathrm{GGN}) \leq 23$ repeats $(P=.014)$. Penile discomfort was less frequent for long $(\mathrm{GGN})>23$ than for medium to short $(G G N) \leq 23$ repeats $(34.6 \%, P=.05)$. Less pubic hair had an OR equal to 8.8 compared with short $(\mathrm{GGN})<23(62.5 \%)$ and medium (GGN)23 (15.9\%), and a OR equal to 8.0 vs medium to long $(\mathrm{GGN}) \geq 23$ repeats $(17.2 \%, P=.012)$. All eight subjects $(100 \%)$ with short $(\mathrm{GGN})<23$ repeats reported the loss of perineal fullness vs $57.1 \%$ of subjects with long $(\mathrm{GGN})>23$ repeats $(P=.05)$. Loss of muscle tone was reported by $87.5 \%$ of short $(\mathrm{GGN})<23$ vs $45.5 \%$ of medium (GGN) 23 carriers $(P=.05)$. Moreover, skin dryness was more commonly reported $(87.5 \%)$ in the short $(\mathrm{GGN})<23$ than in the medium $(\mathrm{GGN}) 23$ $(\mathrm{OR}=21)$, long $(\mathrm{GGN})>23(\mathrm{OR}=12.6)$, and medium to long $(\mathrm{GGN}) \geq 23(\mathrm{OR}=18.4)$ subgroups. Onset of symptoms soon after finasteride discontinuation occurred more frequently in subjects with long $(\mathrm{GGN})>23$ (28.6\%) vs medium (GGN) 23 $(4.5 \%, \mathrm{OR}=8.4)$ and medium to short $(\mathrm{GGN}) \leq 23(5.7 \%$, $\mathrm{OR}=6.4)$ repeats.

\section{DISCUSSION}

In this study, we examined for the first time in detail whether specific symptoms experienced by men with PFS were related to the length of two trinucleotide repeats polymorphic sites located in the large exon 1 of the X-linked $A R$ gene. The AR belongs to the steroid hormone receptor superfamily and mediates androgen functions. ${ }^{16}$ However, only a few studies have investigated $(\mathrm{CAG}) \mathrm{n}$ repeat length in relation to male sexual functions. Recently, Tirabassi et $\mathrm{al}^{30}$ evaluated sexual function recovery after testosterone replacement therapy in subjects with hypogonadism and found that the $A R$ CAG triplet number was negatively associated with changes in improved erectile function, sexual desire, intercourse satisfaction, and total score on the 15-item International Index of Erectile Function after therapy. One study found a significant association of longer (CAG)n with higher total AMS scores in outpatients with sexual and aging male symptoms and patients with psychosomatic or psychiatric disorders but not in healthy control men older than 50 years. ${ }^{31}$

CAG and GGN polymorphisms were supposed to have a role in AGA, but a meta-analysis exploring the association of (CAG) $n$ and (GGN)n with AGA reported no significant findings. ${ }^{32}$

In our study, (CAG)n repeats spanned from 9 to 37; therefore, none of the subjects had at least 40 repeats, a high value typically 
Table 4. Comparison of subgroups of patients with post-finasteride syndrome according to $A R$ gene (GGN)n, short, medium, and long repeats*

\begin{tabular}{|c|c|c|c|c|c|c|c|c|}
\hline & $\begin{array}{l}(\mathrm{GGN})<23 \\
\text { repeats, } \\
\text { short } \\
(\mathrm{n}=8)\end{array}$ & $\begin{array}{l}(\mathrm{GGN}) 23 \\
\text { repeats, } \\
\text { medium } \\
(\mathrm{n}=44)\end{array}$ & $\begin{array}{l}(\mathrm{GGN})>23 \\
\text { repeats, } \\
\text { long } \\
(\mathrm{n}=14)\end{array}$ & $\begin{array}{l}\text { GGN short vs } \\
\text { medium, OR } \\
(95 \%(1), P \text { value }\end{array}$ & $\begin{array}{l}\text { GGN long vs medium, } \\
\text { OR }(95 \% \mathrm{CI}), P \text { value }\end{array}$ & $\begin{array}{l}\text { GGN short vs long, } \\
\text { OR }(95 \% \mathrm{CI}), \\
P \text { value }\end{array}$ & $\begin{array}{l}\text { GGN short vs } \\
\text { medium + long, } \\
\text { OR }(95 \% \mathrm{CI}) \\
P \text { value }\end{array}$ & $\begin{array}{l}\text { GGN long vs medium } \\
+ \text { short, OR }(95 \% \mathrm{CI}), \\
P \text { value }\end{array}$ \\
\hline $\begin{array}{l}\text { Loss of scrotal } \\
\text { sensitivity }\end{array}$ & $6(75.0)$ & $30(68.2)$ & 5 (35.7) & $\begin{array}{l}1.40(0.25-7.83) \\
1.00\end{array}$ & $\begin{array}{l}0.26(0.07-0.92), \\
.031^{\ddagger}\end{array}$ & $\begin{array}{l}5.40(0.78-37.5), \\
.18\end{array}$ & $\begin{array}{l}1.97(0.37-10.6) \\
.70\end{array}$ & $\begin{array}{l}0.25(0.07-0.85) \\
.031^{\ddagger}\end{array}$ \\
\hline Scrotal discomfort & $2(25.0)$ & $15(34.1)$ & $0(-)$ & $\begin{array}{l}0.64(0.12-3.59) \\
1.00\end{array}$ & $0.012^{\dagger, \ddagger}$ & $0.12^{\dagger}$ & $\begin{array}{l}0.96(0.17-5.26) \\
1.00\end{array}$ & $0.014^{t, \ddagger}$ \\
\hline Penile discomfort & $3(37.5)$ & $15(34.1)$ & $1(7.1)$ & $\begin{array}{l}1.16(0.24-5.53) \\
1.00\end{array}$ & $\begin{array}{l}0.15(0.02-1.25) \\
.08^{5}\end{array}$ & $\begin{array}{l}7.80(0.65-93.8) \\
.12\end{array}$ & $\begin{array}{l}1.57(0.34-7.37), \\
.68\end{array}$ & $\begin{array}{l}0.14(0.02-1.20) \\
.05^{\ddagger}\end{array}$ \\
\hline Less pubic hair & $5(62.5)$ & 7 (15.9) & $3(21.4)$ & $\begin{array}{l}8.81(1.70-45.6), \\
.011^{\ddagger}\end{array}$ & $\begin{array}{l}1.44(0.32-6.53) \\
.69\end{array}$ & $\begin{array}{l}6.11(0.90-41.6) \\
.08^{5}\end{array}$ & $\begin{array}{l}8.00(1.64-39.0) \\
.012^{\ddagger}\end{array}$ & $\begin{array}{l}0.91(0.22-3.80) \\
1.00\end{array}$ \\
\hline $\begin{array}{l}\text { Loss of perceived } \\
\text { perineal fullness }\end{array}$ & 8 (100) & 31 (70.5) & 8 (57.1) & $0.18^{\dagger}$ & $\begin{array}{l}0.56(0.16-1.93), \\
.51\end{array}$ & $0.05^{t, \neq, 5}$ & $0.09^{t, 5}$ & $\begin{array}{l}0.44(0.13-1.52) \\
.20\end{array}$ \\
\hline $\begin{array}{l}\text { Loss of muscle } \\
\text { tone }\end{array}$ & 7 (87.5) & 20 (45.5) & 7 (50.0) & $\begin{array}{l}8.40(0.95-74.1), \\
.05^{\ddagger}\end{array}$ & $\begin{array}{l}1.20(0.36-4.00) \\
.77\end{array}$ & $\begin{array}{l}7.00(0.67-72.9) \text {, } \\
.17\end{array}$ & $\begin{array}{l}8.04(0.93-69.5), \\
.055^{5}\end{array}$ & $\begin{array}{l}0.93(0.28-3.01) \\
.90\end{array}$ \\
\hline $\begin{array}{l}\text { Increased skin } \\
\text { dryness }\end{array}$ & 7 (87.5) & $11(25.0)$ & 5 (35.7) & $\begin{array}{l}21.0(2.32-190), \\
.001^{\ddagger}\end{array}$ & $\begin{array}{l}1.67(0.46-6.05) \\
.50\end{array}$ & $\begin{array}{l}12.6(1.19-134), \\
.031^{\ddagger}\end{array}$ & $\begin{array}{l}18.4(2.09-161), \\
.002^{\ddagger}\end{array}$ & $\begin{array}{l}1.05(0.31-3.60) \\
1.00\end{array}$ \\
\hline $\begin{array}{l}\text { Onset of } \\
\text { symptoms } \\
\text { after finasteride } \\
\text { use }\end{array}$ & 1 (12.5) & $2(4.5)$ & $4(28.6)$ & $\begin{array}{l}3.50(0.27-44.7) \\
.36\end{array}$ & $\begin{array}{l}8.40(1.34-52.5), \\
.026^{\ddagger}\end{array}$ & $\begin{array}{l}0.42(0.04-4.66), \\
.62\end{array}$ & $\begin{array}{l}1.44(0.15-14.1) \\
.57\end{array}$ & $\begin{array}{l}6.40(1.24-33.1) \\
.034^{\ddagger}\end{array}$ \\
\hline
\end{tabular}

$\mathrm{OR}=$ odds ratio.

*Dichotomous variables were reported as number (percentage) and $\mathrm{OR}(95 \% \mathrm{CI})$ and $P$ values. $P$ values were evaluated by two-tailed Pearson $\chi^{2}$ or Fisher exact test, as appropriate.

${ }^{\dagger} \mathrm{OR}(\mathrm{CI})$ could not be determined because one group contained no subjects $(0 \%)$ or all subjects $(100 \%)$ for the specific variable.

‡Significant values.

${ }^{\S}$ Nearly significant. 
found in Kennedy disease. ${ }^{20}$ In our patients with PFS, short $(\mathrm{CAG}) \mathrm{n}$ repeats showed a greater decrease in sexual desire and libido (AMS item 17) than medium repeats, whereas long (CAG) $\mathrm{n}$ repeats were associated with worse orgasm satisfaction (ASEX item 5) than short repeats.

Furthermore, subjects with short (CAG)n repeats had scrotal discomfort and increased sperm density less frequently than those with medium or medium to long repeats. Curiously, involuntary muscle spasms were more frequent in the long (CAG)n than in the medium $(\mathrm{OR}=6.3)$ and medium to short $(\mathrm{CAG})<25$ $(\mathrm{OR}=5.5)$ subgroups.

In our study, body mass index did not differ according to (CAG)n subgroup. However, there were more subjects with PFS reporting increased weight after finasteride discontinuation in the long $(\mathrm{CAG}) \mathrm{n}$ than in the medium $(\mathrm{OR}=4.9)$ group. It is worth noting that Corona et $\mathrm{al}^{27}$ associated low testosterone with increased fat mass. Moreover, Tirabassi et al ${ }^{18,33}$ observed that of men with hypogonadotropic hypogonadism, those with shorter $(\mathrm{CAG}) \mathrm{n}$ repeat length had a greater metabolic improvement (including decreased body weight) in response to testosterone replacement therapy. Therefore, it seems plausible that subjects with PFS have decreased androgen levels and that those subjects with long $(\mathrm{CAG}) \mathrm{n}$ repeats were more susceptible to increased body weight.

However, our subjects with PFS and short (CAG)9-19 repeats used finasteride for a shorter period than those with medium to long $(\mathrm{CAG}) \geq 20$ repeats. Moreover, almost all (CAG) 9-19 carriers $(15$ of $18,83.3 \%)$ reported a severe decrease in sexual desire or libido as indicated by a score of 5 on AMS item 17. Further studies are necessary to assess whether finasteride induces more rapidly developing sexual toxic effects in short (CAG)n carriers.

We observed an interesting U-profile for increased skin dryness; the medium (CAG)20-24 group had the lowest frequency, whereas the short $(\mathrm{OR}=4.3)$ and long $(\mathrm{OR}=7.5)(\mathrm{CAG}) \mathrm{n}$ groups had higher frequencies. Remarkably, skin dryness was reported by users of finasteride against hirsutism ${ }^{34}$ and by users of spironolactone, a drug used to treat hormonal acne by inhibiting the AR. ${ }^{11}$ Androgens modulate the rate of cell turnover in the basal layer of the epidermis, the size and activity of the sebaceous glands, the quality of sebaceous secretions, the rate of hair growth, and stimulation of collagen production. ${ }^{11,35} \mathrm{AR}$ is present in fibroblasts and keratinocytes of human skin. One study found the highest androgen binding capacity in cytosol of skin cells from the external genitalia and a lower capacity in pubic skin. ${ }^{36}$ Further investigations are required to explore the role of extreme length of (CAG)n repeats in individuals susceptibility to develop skin dryness after drug treatments. Our findings of a curvilinear U-profile concur with an in vitro study showing that ARs containing short and long (CAG)n stretches, respectively, displayed lower activity than the AR of median (CAG) 22 repeats. ${ }^{37}$ The rationale of an U-profile is that AR is optimally functional with a medium length of the polyglutamine (and/or poly-glycine) stretch. Longer or shorter amino acid repeat stretches could modify optimal protein folding, leading to a suboptimal activity of the receptor protein. ${ }^{6,17,23,38,39}$ By extrapolation, this suggests that skin dryness after finasteride use might be due to decreased AR activity. Interestingly, a metaanalysis showed a non-linear association between $A R$ CAG repeat length and risk of male subfertility. ${ }^{38}$

In this study, subjects with PFS and long (GGN) $>23$ repeats had a better condition regarding physical exhaustion or lacking vitality, depressive mood, and the feeling of passing one's peak than those with medium (GGN)23 repeats. In addition, subjects with PFS and long (GGN)>23 repeats less frequently had loss of scrotal sensitivity and scrotal discomfort but more frequently reported the onset of symptoms after finasteride discontinuation than those with medium (GGN)23 repeats.

Short $(\mathrm{GGN})<23$ carriers showed a better profile concerning need for sleep and/or often feeling tired than medium (GGN)23 carriers but were much more likely to report less pubic hair $(\mathrm{OR}=8.8)$, loss of muscle tone $(\mathrm{OR}=8.4)$, and increased skin dryness $(\mathrm{OR}=21)$ than medium $(\mathrm{GGN}) 23$ carriers.

Overall, we found that the $A R$ polymorphisms can affect several symptoms of PFS. To our knowledge, such specific associations have not been explored previously, which is the reason we cannot compare our results with those of other studies.

Our data showed that short and long (CAG)n and (GGN)n repeats correlate in an unpredictable way with several conditions related to male androgenicity; this could derive from the vast array of genes that are up- or down-modulated by the AR. ${ }^{16,40,41}$ The (CAG)n and (GGN)n polymorphisms also are likely to be in linkage disequilibrium with other polymorphic sites in the human genome, which in turn might determine the observed effects. ${ }^{40}$ In line with observations made by other investigators, ${ }^{39}$ the present findings suggest that the general belief associating long $(C A G) n$ and $(G G N) n$ repeats with a worse androgenic condition $^{18}$ should be taken with caution. ${ }^{39}$ In general, investigations on the influence of $A R$ polymorphisms on specific clinical features (ie, metabolic profile, bone density, and body composition) typically show very contradictory results. ${ }^{18,33}$ This could be due to the concomitant effect of AR-related genetic cofactors (apart from the two studied polymorphisms), which have not been fully explored thus far and which can exert an independent effect on clinical outcomes. ${ }^{40,41}$ It should be noted at this point that the dimension of complexity of the regulation and activities of nuclear receptors is being realized, and that tissue-specific effects and epigenetic changes occurring in a single individual can modulate the action of receptors. ${ }^{41,42}$ Therefore, many more investigations are needed to disclose the biological pathways relating molecular findings to phenotypes. ${ }^{41,43}$

In our study, we decided to go beyond a simple categorization of (CAG)n and (GGN)n repeats as binary (ie, long or short), and we looked at three categories-short, medium, and long repeats - to obtain a better assessment of length variations relating to patients' conditions. Indeed, U-profiles 
have been observed in vitro, ${ }^{23,37}$ and a recent study reported that some markers of male reproductive function in fertile men show a curvilinear association with the CAG or GGN repeat length. ${ }^{39}$ To our knowledge, ours is the first clinical study showing that a U-profile for $(\mathrm{CAG}) \mathrm{n}$ repeats is detectable for a specific human symptom (ie, increased skin dryness after finasteride use). However, it should be noted that for most of the examined symptoms, only one extreme of (CAG) $\mathrm{n}$ and/or $(\mathrm{GGN}) \mathrm{n}$ repeats behaved differently from the medium length subgroup.

A limitation of this study is the limited number of subjects. Larger studies are warranted to substantiate the present finding in white and other ethnic groups. Larger investigations also could assess haplotypes comprising all the possible combinations of short, medium, and long (CAG)n plus short, medium, and long (GGN)n repeats. A general limitation of this study work is that some symptoms reported by patients with PFS could not be objectively determined. Furthermore, the retrospective design of our study did not allow a clinical assessment of these men before finasteride use. Future studies are necessary to assess the $A R$ genetic profile and testosterone levels in subjects who developed PFS compared with subjects who did not develop adverse symptoms after using finasteride against AGA.

\section{CONCLUSION}

Causes and predisposing factors responsible for the development of long-term adverse side effects in young men who used low-dose finasteride against AGA remain an enigma. Several symptoms were in common in more than $70 \%$ of patients with PFS, but a plethora of other disturbances was reported by a minority of patients, with some clearly related and some not to androgenicity.

Our study showed that the length of two trinucleotide repeats in the $A R$ gene contribute to the frequency of some specific symptoms reported by patients with PFS. The (CAG)n and (GGN)n polymorphisms were involved in two specific symptoms (ie, scrotal discomfort and increased skin dryness); for other symptoms, only one of the two polymorphisms was involved, which is likely a reflection of the complex modulation of AR activity. $^{16,40}$

Our investigation using a precision medicine approach suggested genetic implications in symptoms of patients with PFS. Much more genetic and non-genetic research is necessary to elucidate the pathophysiologic pathways leading to the onset and persistence of adverse effects in former finasteride users.

\section{ACKNOWLEDGMENTS}

We thank Giorgio Mazzon and Francesco La Marra for helping in subject recruitment. We are very grateful to Blase Billack (St John's University, Queens, NY, USA) for critical revisions of this report, Francesca Migliozzi for reading the report, and to Elena De Mattia for technical support.
Corresponding Author: Sabina Cauci, PhD, Associate Professor, Dipartimento di Scienze Mediche e Biologiche, Università di Udine, Piazzale Kolbe 4, Udine 33100, Italy. Tel: +39-0432-494312; Fax: +39-0432-494301; E-mail: sabina.cauci@uniud.it

Conflicts of Interest: None.

Funding: None.

\section{STATEMENT OF AUTHORSHIP}

\section{Category 1}

(a) Conception and Design

Sabina Cauci; Giovanni Chiriacò; Erika Cecchin; Giuseppe Toffoli; Giuseppe Stinco; Carlo Trombetta

(b) Acquisition of Data

Sabina Cauci; Ciovanni Chiriacò; Erika Cecchin; Giuseppe Toffoli; Serena Xodo; Ciuseppe Stinco; Carlo Trombetta

(c) Analysis and Interpretation of Data

Sabina Cauci; Giovanni Chiriacò; Erika Cecchin; Giuseppe Stinco; Carlo Trombetta

\section{Category 2}

(a) Drafting the Article

Sabina Cauci; Giovanni Chiriacò; Erika Cecchin; Serena Xodo; Ciuseppe Stinco

(b) Revising It for Intellectual Content

Sabina Cauci; Giovanni Chiriacò; Giuseppe Toffoli; Ciuseppe Stinco; Carlo Trombetta

\section{Category 3}

(a) Final Approval of the Completed Article

Sabina Cauci; Giovanni Chiriacò; Erika Cecchin; Giuseppe Toffoli; Serena Xodo; Ciuseppe Stinco; Carlo Trombetta

\section{REFERENCES}

1. Traish AM, Hassani J, Guay AT, et al. Adverse side effects of 5alpha-reductase inhibitors therapy: persistent diminished libido and erectile dysfunction and depression in a subset of patients. J Sex Med 2011;8:872-884.

2. La Marra F, Di Loreto C, Mazzon G, et al. Preliminary evidence of a peculiar hormonal profile in men with adverse effects after use of finasteride against androgenetic alopecia. Am J Pathol 2012;181:S8.

3. Irwig MS, Kolukula S. Persistent sexual side effects of finasteride for male pattern hair loss. J Sex Med 2017;8:1747-1753.

4. Chiriacò G, Cauci S, Mazzon G, et al. An observational retrospective evaluation of 79 young men with long-term adverse effects after use of finasteride against androgenetic alopecia. Andrology 2016;4:245-250.

5. Traish AM, Melcangi RC, Bortolato M, et al. Adverse effects of 5alpha-reductase inhibitors: what do we know, don't know, and need to know? Rev Endocr Metab Disord 2015;16:177198.

6. Cecchin E, De Mattia E, Mazzon G, et al. A pharmacogenetic survey of androgen receptor (CAG)n and (GGN)n polymorphisms in patients experiencing long term side effects 
after finasteride discontinuation. Int J Biol Markers 2014; 29:e310-e316.

7. Belknap SM, Aslam I, Kiguradze T, et al. Adverse event reporting in clinical trials of finasteride for androgenic alopecia: a meta-analysis. JAMA Dermatol 2015;151:600-606.

8. Yamana K, Labrie F, Luu-The V. Human type 3 5alphareductase is expressed in peripheral tissues at higher levels than types 1 and 2 and its activity is potently inhibited by finasteride and dutasteride. Horm Mol Biol Clin Investig 2010;2:293-299.

9. Fouad Mansour M, Pelletier M, Tchernof A. Characterization of 5alpha-reductase activity and isoenzymes in human abdominal adipose tissues. J Steroid Biochem Mol Biol 2016; 161:45-53.

10. Irwig MS. Safety concerns regarding 5alpha reductase inhibitors for the treatment of androgenetic alopecia. Curr Opin Endocrinol Diabetes Obes 2015;22:248-253.

11. Lai JJ, Chang P, Lai KP, et al. The role of androgen and androgen receptor in skin-related disorders. Arch Dermatol Res 2012;304:499-510.

12. Mella JM, Perret MC, Manzotti M, et al. Efficacy and safety of finasteride therapy for androgenetic alopecia: a systematic review. Arch Dermatol 2010;146:1141-1150.

13. Liu L, Zhao S, Li F, et al. Effect of Salpha-reductase inhibitors on sexual function: a meta-analysis and systematic review of randomized controlled trials. J Sex Med 2016;13:1297-1310.

14. Di Loreto C, La Marra F, Mazzon G, et al. Immunohistochemical evaluation of androgen receptor and nerve structure density in human prepuce from patients with persistent sexual side effects after finasteride use for androgenetic alopecia. PLoS One 2014;9:e0100237.

15. Gottlieb B, Beitel LK, Nadarajah A, et al. The androgen receptor gene mutations database: 2012 update. Hum Mutat 2012;33:887-894.

16. Simental JA, Sar M, Lane MV, et al. Transcriptional activation and nuclear targeting signals of the human androgen receptor. J Biol Chem 1991;266:510-518.

17. Casella R, Maduro MR, Lipshultz LI, et al. Significance of the polyglutamine tract polymorphism in the androgen receptor. Urology 2001;58:651-656.

18. Tirabassi G, Cignarelli A, Perrini S, et al. Influence of CAG repeat polymorphism on the targets of testosterone action. Int J Endocrinol 2015;2015:1-12.

19. La Spada AR, Wilson EM, Lubahn DB, et al. Androgen receptor gene mutations in $\mathrm{X}$-linked spinal and bulbar muscular atrophy. Nature 1991;352:77-79.

20. Lumbroso S, Lobaccaro JM, Vial C, et al. Molecular analysis of the androgen receptor gene in Kennedy's disease. Report of two families and review of the literature. Horm Res 1997; 47:23-29.

21. Pan B, Li R, Chen Y, et al. Genetic association between androgen receptor gene CAG repeat length polymorphism and male infertility: a meta-analysis. Medicine (Baltimore) 2016; 95:e2878.
22. Sun JH, Lee SA. Association between CAG repeat polymorphisms and the risk of prostate cancer: a meta-analysis by race, study design and the number of (CAG)n repeat polymorphisms. Int J Mol Med 2013;32:1195-1203.

23. Lundin KB, Giwercman A, Dizeyi $N$, et al. Functional in vitro characterisation of the androgen receptor GGN polymorphism. Mol Cell Endocrinol 2007;264:184-187.

24. Jiang W, Zhang J, Zhou Q, et al. Predictive value of GGN and CAG repeat polymorphisms of androgen receptors in testicular cancer: a meta-analysis. Oncotarget 2016;7:13754-13764.

25. McGahuey CA, Gelenberg AJ, Laukes CA, et al. The Arizona Sexual Experience Scale (ASEX): reliability and validity. J Sex Marital Ther 2000;26:25-40.

26. Heinemann LA, Moore C, Dinger JC, et al. Sensitivity as outcome measure of androgen replacement: the AMS scale. Health Qual Life Outcomes 2006;4:23.

27. Corona G, Giagulli VA, Maseroli E, et al. Therapy of endocrine disease: testosterone supplementation and body composition: results from a meta-analysis study. Eur J Endocrinol 2016; 174:R99-R116.

28. Bhopal R, Donaldson L. White, European, western, Caucasian, or what? Inappropriate labeling in research on race, ethnicity, and health. Am J Public Health 1998;88:1303-1307.

29. Corona G, Rastrelli G, Vignozzi L, et al. How to recognize lateonset hypogonadism in men with sexual dysfunction. Asian J Androl 2012;14:251-259.

30. Tirabassi G, Corona G, Biagioli A, et al. Influence of androgen receptor CAG polymorphism on sexual function recovery after testosterone therapy in late-onset hypogonadism. J Sex Med 2015;12:381-388.

31. Schneider G, Nienhaus K, Gromoll J, et al. Aging males' symptoms in relation to the genetically determined androgen receptor CAG polymorphism, sex hormone levels and sample membership. Psychoneuroendocrinology 2010;35:578-587.

32. Zhuo FL, Xu W, Wang $L$, et al. Androgen receptor gene polymorphisms and risk for androgenetic alopecia: a metaanalysis. Clin Exp Dermatol 2012;37:104-111.

33. Tirabassi G, delli Muti N, Corona G, et al. Androgen receptor gene CAG repeat polymorphism regulates the metabolic effects of testosterone replacement therapy in male postsurgical hypogonadotropic hypogonadism. Int J Endocrinol 2013;2013:1-7.

34. van Zuuren EJ, Fedorowicz Z, Carter B, et al. Interventions for hirsutism (excluding laser and photoepilation therapy alone). Cochrane Database Syst Rev 2015;(4):CD010334.

35. Barrault C, Garnier J, Pedretti N, et al. Androgens induce sebaceous differentiation in sebocyte cells expressing a stable functional androgen receptor. J Steroid Biochem Mol Biol 2015;152:34-44.

36. Mestayer C, Berthaut I, Portois MC, et al. Predominant expression of 5 alpha-reductase type 1 in pubic skin from normal subjects and hirsute patients. Clin Endocrinol Metab 1996;81:1989-1993.

37. Nenonen H, Bjork C, Skjaerpe PA, et al. CAG repeat number is not inversely associated with androgen receptor activity in vitro. Mol Hum Reprod 2010;16:153-157. 
38. Nenonen HA, Giwercman A, Hallengren $E$, et al. Non-linear association between androgen receptor CAG repeat length and risk of male subfertility-a meta-analysis. Int $J$ Androl 2011; 34:327-332.

39. Brokken $L J$, Rylander $L$, Jonsson $B A$, et al. Non-linear association between androgen receptor CAG and GGN repeat lengths and reproductive parameters in fertile European and Inuit men. Mol Cell Endocrinol 2013;370:163-171.

40. van de Wijngaart $D J$, Dubbink $H J$, van Royen $M E$, et al. Androgen receptor coregulators: recruitment via the coactivator binding groove. Mol Cell Endocrinol 2012;352:57-69.

41. Shukla GC, Plaga AR, Shankar E, et al. Androgen receptorrelated diseases: what do we know? Andrology 2016; $4: 366-381$.
42. delli Muti N, Tirabassi G, Buldreghini E, et al. Synergistic effect of androgen receptor (CAG repeat length) and endothelial nitric oxide synthase (Clu298Asp variant) gene polymorphisms on seminal parameters in men with idiopathic oligo-asthenozoospermia. Endocrine 2014;47:322-324.

43. Ahmed SF, Cheng A, Dovey $L$, et al. Phenotypic features, androgen receptor binding, and mutational analysis in 278 clinical cases reported as androgen insensitivity syndrome. J Clin Endocrinol Metab 2000;85:658-665.

\section{SUPPLEMENTARY DATA}

Supplementary data related to this article can be found at http://dx.doi.org/10.1016/j.esxm.2016.11.001. 